\title{
Influences of heel height on human postural stability and functional mobility between inexperienced and experienced high heel shoe wearers
}

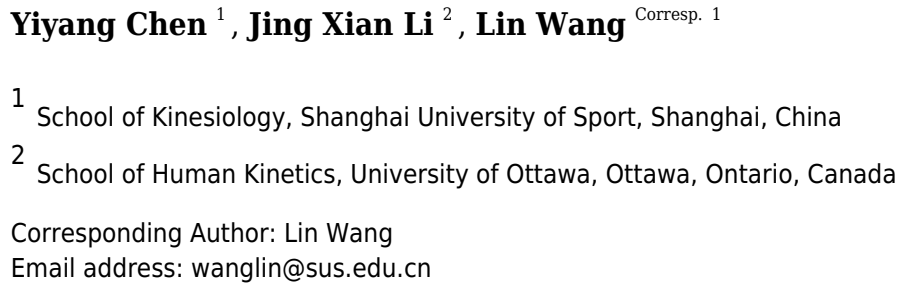

Background. High heel shoes (HHS) can affect human postural control because elevated heel height $(\mathrm{HH})$ may result in plantar flexed foot and limit ankle joint range of motion during walking. Effects of $\mathrm{HH}$ and $\mathrm{HHS}$ wearing experience on postural stability during selfinitiated and externally triggered perturbations are less examined in the literature. Hence, the objective of the present study is to investigate the influences of $\mathrm{HH}$ on human postural stability during dynamic perturbations, perceived stability, and functional mobility between inexperienced and experienced HHS wearers. Methods. A total of 41 female participants were recruited ( 21 inexperienced HHS wearers and 20 experienced HHS wearers). Sensory organization test (SOT), motor control test (MCT), and limits of stability (LOS) were conducted to measure participant's postural stability by using computerized dynamic posturography. Functional reach test and timed up and go test were performed to measure functional mobility. The participants' self-perceived stability was assessed by visual analog scale. Four pairs of shoes with different $\mathrm{HH}$ (i.e., 0.8, 3.9, 7.0, and $10.1 \mathrm{~cm}$ ) were applied to participants randomly. Repeated measures analysis of variance was conducted to detect the effects of $\mathrm{HH}$ and HHS wearing experience on each variable. Results. During selfinitiated perturbations, equilibrium score remarkably decreased when wearing $10.1 \mathrm{~cm}$ compared with flat shoes and $3.9 \mathrm{~cm}$ HHS. The contribution of vision to postural stability was larger in $10.1 \mathrm{~cm}$ HHS than in flat shoes. The use of ankle strategy worsened when $\mathrm{HH}$ increased to $7 \mathrm{~cm}$. Similarly, the directional control of the center of gravity (COG) decreased for $7 \mathrm{~cm}$ HHS in LOS. Experienced wearers showed significantly higher percentage of ankle strategy and COG directional control than novices. Under externally triggered perturbations, postural stability was substantially decreased when $\mathrm{HH}$ reached $3.9 \mathrm{~cm}$ in MCT. No significant difference was found in experienced wearers compared with novices in MCT. Experienced wearers exhibited considerably better functional mobility and perceived stability with increased $\mathrm{HH}$. Conclusions. The use of HHS may worsen dynamic 
postural control and functional mobility when $\mathrm{HH}$ increases to $3.9 \mathrm{~cm}$. Although experienced HHS wearers exhibit higher proportion of ankle strategy and COG directional control, the experience may not influence overall human postural control. Sensory organization ability, ankle strategy and COG directional control might provide useful information in developing a safety system and prevent HHS wearers from falling. 
1 Influences of heel height on human postural stability and functional mobility between

2 inexperienced and experienced high heel shoe wearers

3

4 Yiyang Chen ${ }^{1}$, Jing Xian $\mathrm{Li}^{2}$, and Lin Wang ${ }^{1}$

51 School of Kinesiology, Shanghai University of Sport, Shanghai, China

62 School of Human Kinetics, University of Ottawa, Ottawa, Canada

7

8 Corresponding author:

9 Lin Wang ${ }^{1}$

10188 Hengren Road, Yangpu District, Shanghai, 200438, China

11 E-mail: wanglin@sus.edu.cn 
13

\section{Abstract}

Background. High heel shoes (HHS) can affect human postural control because elevated heel height $(\mathrm{HH})$ may result in plantar flexed foot and limit ankle joint range of motion during walking. Effects of $\mathrm{HH}$ and HHS wearing experience on postural stability during self-initiated and externally triggered perturbations are less examined in the literature. Hence, the objective of the present study is to investigate the influences of $\mathrm{HH}$ on human postural stability during dynamic perturbations, perceived stability, and functional mobility between inexperienced and experienced HHS wearers.

Methods. A total of 41 female participants were recruited (21 inexperienced HHS wearers and 20 experienced HHS wearers). Sensory organization test (SOT), motor control test (MCT), and limits of stability (LOS) were conducted to measure participant's postural stability by using computerized dynamic posturography. Functional reach test and timed up and go test were performed to measure functional mobility. The participants' self-perceived stability was assessed by visual analog scale. Four pairs of shoes with different HH (i.e., 0.8, 3.9, 7.0, and $10.1 \mathrm{~cm}$ ) were applied to participants randomly. Repeated measures analysis of variance was conducted to detect the effects of $\mathrm{HH}$ and HHS wearing experience on each variable.

Results. During self-initiated perturbations, equilibrium score remarkably decreased when wearing $10.1 \mathrm{~cm}$ compared with flat shoes and $3.9 \mathrm{~cm}$ HHS. The contribution of vision to postural stability was larger in $10.1 \mathrm{~cm}$ HHS than in flat shoes. The use of ankle strategy worsened when $\mathrm{HH}$ increased to $7 \mathrm{~cm}$. Similarly, the directional control of the center of gravity (COG) decreased for $7 \mathrm{~cm}$ HHS in LOS. Experienced wearers showed significantly higher percentage of ankle strategy and COG directional control than novices. Under externally triggered perturbations, postural stability was substantially decreased when $\mathrm{HH}$ reached $3.9 \mathrm{~cm}$ in MCT. No significant difference was found in experienced wearers compared with novices in MCT. Experienced wearers exhibited considerably better functional mobility and perceived stability with increased $\mathrm{HH}$.

Conclusions. The use of HHS may worsen dynamic postural control and functional mobility 
40

41

42

43

when $\mathrm{HH}$ increases to $3.9 \mathrm{~cm}$. Although experienced HHS wearers exhibit higher proportion of ankle strategy and COG directional control, the experience may not influence overall human postural control. Sensory organization ability, ankle strategy and COG directional control might provide useful information in developing a safety system and prevent HHS wearers from falling. Keywords. high heel shoes; heel height; wearing experience; postural stability; functional mobility

\section{Introduction}

High heel shoes (HHS) have been widely used among women in several centuries; $37 \%$ to $69 \%$ of women wear HHS daily (American Podiatric Medical Association, 2003). HHS are featured with heel evaluation, rigid heel cap and curved plantar region, which interfere with natural foot motion (Cronin, 2014). A more plantar flexed and supinated foot position can alter the distribution of plantar pressure, affect muscle activities around ankle joints, and limit the range of motion (ROM) of the ankle during standing and walking (Ko et al., 2009; Luximon et al., 2015; Simonsen et al., 2012). A number of studies have documented that the effects of HHS are not localized to the ankle; instead, a "chain reaction" of kinematic effects travels up the lower limb and disturbs the displacement of the center of mass (COM) (Chien et al., 2013; Cronin, 2014; Schroeder \& Hollander, 2018). These biomechanical alterations can decrease perceived stability, impair postural control, and increase the risks of falling among HHS wearers (Luximon et al., 2015; Wan et al., 2019). The rate of high heels-related injuries increased from $7.1 \%$ to $14.1 \%$ during the 11-year period from 2002 to 2012. Most of the injuries were sprains or strains occurred to either the ankle or foot body regions (Barnish \& Barnish, 2009; Moore et al., 2015).

One of the risk factors on high heels-related injuries is decreased postural stability among HHS wearers (Wan et al., 2019). Postural control is the ability to stabilize and restore the body's COM relative to the base of support (BOS) during self-initiated and externally triggered perturbations (Horak, 2006; Winter, 1995). To maintain postural stability, a complex motor skill based on the interaction of proprioceptive, visual, and vestibular system is utilized in this process 
67 (Mancini \& Horak, 2010). Wearing HHS can cause biomechanical constrains and disturb human movement strategies through reduced BOS and elevated heel height (HH) (Chien et al., 2013). The HHS wearers tend to apply different movement strategies (e.g., ankle and hip strategy) to maintain the stability of the body's equilibrium with regard to elevated $\mathrm{HH}$ during standing, walking, and dynamic perturbations.

A number of studies found that different $\mathrm{HH}$ can influence postural stability through interfering with the stabilization of COM with respect to the BOS. Different sensory and movement strategies are also involved in the process of postural control in HHS wearers. Recent studies have examined that HHS wearers had significantly worse standing balance starting at 7 $\mathrm{cm} \mathrm{HH}$ by analyzing the center of pressure (COP) magnitude in quiet stance and limits of stability test (LOS) (Choi \& Cho, 2006; Gerber et al., 2012; Mika et al., 2016). During extrinsic perturbations, previous studies demonstrated that HHS can impair human balance (e.g., sinusoidal oscillations and waist pulling) (Choi \& Cho, 2006; Sun et al., 2017). When HH increased to $10 \mathrm{~cm}$, increased use of ankle strategy, slow center of gravity (COG) movement velocity, and decreased body equilibrium were observed with increased HH (Hapsari \& Xiong, 2016; Truszczyńska et al., 2019). However, no difference in the interaction of sensory systems was found in postural control among HHS wearers with increased HH (Hapsari \& Xiong, 2016). It will be worthwhile to detect how sensory systems interact during postural control, and to what extend can $\mathrm{HH}$ affect movement strategy and influence human overall postural control accordingly.

HHS experience might be another vital factor that can influence HHS wearers' postural stability as well. Previous research has shown significant muscular alterations, such as overwork muscle activities in medial gastrocnemius and peroneus longus, shortened calf muscles, and increased Achilles tendon stiffness after long-term use of HHS (Cronin et al., 2012; Csapo et al., 2010; Kermani et al., 2018). These muscular accommodations around ankle joints can affect the efficient use of ankle strategies to return the body to equilibrium during standing (Chien et al., 2014; Rahimi et al., 2017; Wan et al., 2019). However, Xiong and Hapsari found no significant 
94

95

96

difference in self-initiated standing balance and functional mobility between experienced HHS wearers and inexperienced HHS wearers, although the experienced group showed higher directional control of COG in LOS (Hapsari \& Xiong, 2016). Therefore, whether HHS wearing experience can influence human postural stability and functional mobility remains unclear.

Hence, the current study aims to investigate the effects of $\mathrm{HH}$ (i.e., $0.8,3.9,7.0$, and $10.1 \mathrm{~cm}$ ) and HHS experience on postural stability during dynamic perturbations, perceived stability, and functional mobility in women. We hypothesized that human postural stability could decrease with increasing $\mathrm{HH}$, and HHS experience could improve performance in postural control and functional mobility test.

\section{Materials \& Methods}

\subsection{Participants}

A total of 41 female participants were recruited from the local university and communities (21 inexperienced HHS wearers and 20 experienced HHS wearers). All participants had a shoe size of EU 36-39 and self-reported to be free from lower limb injuries for a minimum of six months prior to the study. Participants with any history of musculoskeletal, cardiovascular, neurological, and vestibular abnormalities were excluded from the experiment. Anthropometrics were measured prior to the experiment (i.e., body height, weight, foot length, and arch height). The measurements of foot length and arch height were taken under two conditions: $10 \%$ and 90\% weightbearing loads (Zifchock et al., 2017). Arch height flexibility (AHF) was defined as the changes in arch height from $10 \%$ to $90 \%$ weightbearing conditions, normalized to $80 \%$ body weight. Experienced HHS wearers were those who had worn narrow-heeled shoes with a minimum $\mathrm{HH}$ of $4 \mathrm{~cm}$ more than twice per week and at least eight hours per day for one year. Inexperienced HHS wearers were participants wearing HHS less than once per week (Hapsari \& Xiong, 2016; Wan et al., 2019). The study was approved by the ethics committee of Shanghai University of Sport (Number: 2018074), and all subjects were provided written consents prior to the experiment. 
120

121

122

123

124

125

126

127

128

129

130

131

132

133

134

135

136

137

138

139

140

141

142

143

144

145

146

\subsection{Experimental shoes}

Experimental shoes with $\mathrm{HH}$ of $0.8,3.9,7.0$, and $10.1 \mathrm{~cm}$ were used in the study (Figure 1). All the experimental shoes were manufactured by the same manufacturer. The shoe style and materials were maintained the same to minimize confounding variance. Except for the $0.8 \mathrm{~cm}$ HHS as the baseline condition, the three other types of HHS were featured with narrow-heeled shoes $(12.5 \mathrm{~mm} * 12.0 \mathrm{~mm})$. Participants were allowed to familiarize themselves with the most suitable experimental shoes with shoe size ranging from EU 36-39 prior to the experiment. The four HHS testing conditions were assigned to participants in random order.

Insert Figure 1 here

\subsection{Data collection}

\subsubsection{Postural control}

NeuroCom Balance Manager System (Version 9.3, Natus Medical Incorporated, USA) SMART EquiTest was used to assess postural stability by measuring the participants' COG alignment at a sampling frequency of $100 \mathrm{~Hz}$ after they were familiar with the experimental HHS (Chander et al., 2016; Hapsari \& Xiong, 2016). Computerized dynamic posturography has been proven to be a "gold standard" for assessing postural stability with high reliability and validity (Harro \& Garascia, 2019). Prior to the test, participants were secured with a protective vest from falling off the instrumentation. They were instructed to stand on the two force plates $(23 \mathrm{~cm} * 46$ $\mathrm{cm})$ with feet aligned with the platform axis as the initial position. SOT and LOS were used to test the participants' standing balance during self-initiated perturbations, whereas postural stability during externally triggered perturbations was tested by motor control test (MCT). Participants were asked to stand still with their feet fixed in the initial position. A five-minute rest was allowed between three tests to prevent potential fatigue.

\subsubsection{Sensory organization test (SOT)}

SOT utilizes the sway-referencing capabilities of the visual surroundings and the support surface to evaluate the integration of the sensory systems in postural control by selectively disrupting somatosensory and/or visual information. Moderate to excellent reliability has been 
147

148

149

150

151

152

153

154

155

156

157

158

159

160

161

162

163

164

165

166

167

168

169

170

171

172

173

established in SOT among healthy adults (Ford-smith et al., 1995; Harro \& Garascia, 2019;

Tsang et al., 2004), and among patients with multiple sclerosis (Hebert \& Manago, 2017)and transtibial amputation (Jayakaran et al., 2011). The six testing conditions in SOT are described in Table 1 (Yin \& Wang, 2020). Each testing condition was repeated three times. All the testing orders were randomly assigned to the participants (Dickin, 2010). The equilibrium and composite scores $(0-100)$ represent the ability of the participants to maintain postural stability in each condition and overall postural control, respectively. The strategy scores (0-100) quantify the relative amount of movement about the ankle and hip strategies that participants used in maintaining postural stability. A strategy score approaching 100 indicates that ankle strategy is more dominant in maintaining balance, whereas a score closest to 0 suggests that the participant uses hip strategy dominantly to stabilize her body under each trial. Somatosensory (SOM), vestibular (VEST), and visual scores (VIS) (0-100) in sensory analysis quantify the participants' ability to integrate proprioception, vestibulum, and vision information that contribute to balance, respectively.

\section{Insert Table 1 here}

\subsubsection{Motor control test (MCT)}

Postural stability under support surface perturbations was assessed by MCT (Figure 2). The two force plates with translation capabilities in backward and forward directions can create six perturbing conditions which are small backward translation (SBT), medium backward translation (MBT), large backward translation (LBT), small forward translation (SFT), medium forward translation (MFT), and large forward translation (LFT). Each testing condition was repeated three times. The six testing conditions were assigned in random order. The displacement of the support surface is scaled to the participant's height during each translation. The outcome measures were composite latency and amplitude scaling. Composite latency measures the reaction time from the initiation of translation of the platform to the displacement of COG in milliseconds. Amplitude scaling is measured for right leg in units of angular momentum and normalized to body height and weight, which quantifies the force generated from the lower limb 
174 in response to the external perturbations (Vanicek et al., 2013).

175

176

177

178

179

180

181

182

183

184

185

186

187

188

189

190

191

192

193

194

195

196

197

198

199

200

Insert Figure 2 here

\subsubsection{Limits of stability test (LOS)}

LOS quantifies the ability of participants to intentionally displace their COG within the BOS. In LOS, a computerized screen was placed in front of the participants. They were instructed to lean their body on the sagittal plane in each direction to reach to the target location displayed on the screen as quick as possible upon hearing an auditory cue. Then, participants were required to remain in that position for $10 \mathrm{~s}$. The outcome measures were COG movement velocity and directional control (DCL). COG movement velocity in degree per second $(\%$ s) represents the average COG movement speed from the initial place to the target position. Directional control was calculated as the amount of the COG movement toward the intended direction minus the amount of off-axis movement (Yin \& Wang, 2020).

\subsubsection{Functional mobility test}

After postural control tests, functional reach test (FRT) and timed up and go test (TUGT) were performed to measure functional mobility. FRT measures the maximum forward reach of the participants. Participants were instructed to lean their body forward as far as possible without stepping or reaching for assistance. Three trials were conducted for data normalization purposes. In TUGT, participants were requested to sit on a standard chair with their back against the chair, arms resting on the chair's arms. They were instructed to walk a $3 \mathrm{~m}$ straight line, make turns, walk back to the chair and sit down. Participants were asked to walk at their comfortable speed. The time between the participants' buttocks leaving and touching the seat surface was recoded. The fastest among the three testing trials was used for data analysis (Schoppen et al., 1999).

\subsubsection{Perceived stability}

Thereafter, the participants were instructed to quantify their perceived stability in FRT on a visual analog scale (VAS). The scores range from 0-100. The VAS score of 0 indicates that the participants were perceived as unstable, whereas a score of 100 suggests the most stable situation that can be perceived.

Peer) reviewing PDF | (2020:07:50629:1:1:NEW 23 Sep 2020) 
201

202

203

204

205

206

207

208

209

210

211

212

213

214

215

216

217

218

219

220

221

222

223

224

225

226

227

228

\subsection{Statistical analysis}

All data were presented as mean \pm standard deviation (SD). The normal distribution of data was examined by the Shapiro-Wilk test. Repeated measurement of ANOVA (HH * HHS wearing experience) was conducted to detect the effects of $\mathrm{HH}$ and $\mathrm{HHS}$ wearing experience on each variable. Simple main effect analysis was used for post hoc comparisons. Significance was set at an alpha level of $p=0.05$. Partial eta-squared $\left(\eta^{2}\right)$ effect size, $95 \%$ confidence interval (CI), and F-statistic were reported. Statistical analysis was performed using SPSS 22.0 statistical software package (SPSS Inc., Chicago, USA).

\section{Results}

\subsection{Demographic characteristics of the participants}

Table 2 illustrates the characteristics of the participants. No significant differences were observed in age, height, weight, body mass index (BMI), foot length 10\% weightbearing, foot length $90 \%$ weightbearing and AHF between the two groups. The experienced group showed significantly higher HHS wearing frequency than the inexperienced group $(p<0.001)$.

Insert Table 2 here

\subsection{SOT}

The descriptive data of SOT are shown in Table 3 . No statistically significant interaction was found between the $\mathrm{HH}$ and HHS wearing experience on the outcome measures of SOT (Table 3). The main effect of $\mathrm{HH}$ was significant for the equilibrium score in $\mathrm{C} 1(\mathrm{~F}(3,38)=8.342$, $\left.p<0.001, \eta^{2}=0.202\right), \mathrm{C} 2\left(\mathrm{~F}(3,38)=14.498, p<0.001, \mathrm{\eta}^{2}=0.202\right), \mathrm{C} 3(\mathrm{~F}(3,38)=10.428, p<0.001$, $\left.\eta^{2}=0.202\right)$, and C5 $\left(\mathrm{F}(3,38)=10.920, p<0.001, \eta^{2}=0.202\right)$. No significant effect of HHS wearing experience was found on the equilibrium score. Post hoc analysis revealed significantly lower equilibrium score in $10.1 \mathrm{~cm}$ than $7 \mathrm{~cm}$ HHS among experienced HHS wearers in $\mathrm{C} 2(p=0.035$, $95 \% \mathrm{CI}=0.143-5.590)$.

The main effect of HH was significant for the strategy score in six conditions $\left(\mathrm{F}(3,38)=12.234, p<0.001, \mathrm{\eta}^{2}=0.176 ; \mathrm{F}(3,38)=29.763, p<0.001, \mathrm{\eta}^{2}=0.271 ; \mathrm{F}(3,38)=21.591, p<\right.$ $0.001, \eta^{2}=0.356 ; \mathrm{F}(3,38)=3.125, p=0.036, \eta^{2}=0.074 ; \mathrm{F}(3,38)=10.598, p<0.001, \mathrm{\eta}^{2}=0.214$; $\left.\mathrm{F}(3,38)=5.601, p=0.002, \eta^{2}=0.126\right)$. The main effect of wearing experience was also significant 
229 in $\mathrm{C} 3\left(\mathrm{~F}(1,40)=10.841, p=0.002, \eta^{2}=0.218\right), \mathrm{C} 5\left(\mathrm{~F}(1,40)=4.977, p=0.032, \eta^{2}=0.022\right)$, and $\mathrm{C} 6$

$230\left(\mathrm{~F}(1,40)=5.857, p=0.020, \eta^{2}=0.132\right)$. The strategy score decreased significantly when $\mathrm{HH}$

231 increased to $7 \mathrm{~cm}$ compared with flat shoes among experienced HHS wearers in C5 ( $p=0.001$,

$23295 \% \mathrm{CI}=0.997-4.036)$. In C3, the experienced HHS wearers demonstrated significantly higher

233 strategy score than inexperienced HHS wearers in flat shoes $(\mathrm{t}=-2.231, p=0.033), 3.9 \mathrm{~cm}$

$234(\mathrm{t}=-2.404, p=0.023)$, and $10.1 \mathrm{~cm}$ HHS $(\mathrm{t}=-3.327, p=0.002$; Table 3$)$.

235 Table 3 illustrates that the main effect of HH was significant for sensory analysis score in $236 \operatorname{SOM}\left(\mathrm{F}(3,38)=3.059, p=0.031, \eta^{2}=0.099\right)$ and $\operatorname{VIS}\left(\mathrm{F}(3,38)=4.270, p=0.010, \eta^{2}=0.099\right)$, but the

237 main effect of wearing experience was undetected. Post hoc analysis showed that the sensory 238 analysis score declined significantly in VIS when wearing $10.1 \mathrm{~cm}$ HHS compared with flat 239 shoes in inexperienced wearers $(p=0.008,95 \% \mathrm{CI}=1.470-12.244)$.

$240 \quad$ Insert Table 3 here

$241 \quad 3.3$ MCT

242 No significant interaction between the $\mathrm{HH}$ and wearing experience was detected on outcome 243 measures of MCT. As shown in Table 4, the main effect of HH was significant for the composite 244 latency $\left(\mathrm{F}(3,38)=3.121, p=0.044, \eta^{2}=0.080\right)$, whereas no significant difference was detected in 245 the pairwise comparison. The HH revealed a significant main effect on amplitude scaling in SBT $246\left(\mathrm{~F}(3,38)=7.004, p<0.001, \eta^{2}=0.163\right), \operatorname{MBT}\left(\mathrm{F}(3,38)=3.630, p=0.015, \eta^{2}=0.092\right), \mathrm{SFT}$

$247\left(\mathrm{~F}(3,38)=15.604, p<0.001, \mathrm{\eta}^{2}=0.302\right), \operatorname{MFT}\left(\mathrm{F}(3,38)=24.919, p<0.001, \eta^{2}=0.409\right)$, and LFT $248\left(\mathrm{~F}(3,38)=9.522, p<0.001, \eta^{2}=0.209\right)$. No significant main effect was investigated for HHS 249 wearing experience on amplitude scaling in six perturbing conditions. In MFT, the amplitude 250 scaling was significantly higher when $\mathrm{HH}$ increased to $7 \mathrm{~cm}$ compared with flat shoes among 251 experienced wearers $(p=0.013,95 \% \mathrm{CI}=-2.193-0.207)$.

252 Insert Table 4 here

\section{$253 \quad 3.4$ LOS}

As shown in Table 5, no statistically significant interaction was found between the $\mathrm{HH}$ and HHS wearing experience on COG movement velocity, whereas the two-way interaction was 256 significant on directional control $\left(\mathrm{F}(3,38)=7.790, p<0.001, \mathrm{\eta}^{2}=0.166\right)$. The main effect of $\mathrm{HH}$ 
257 was significant for COG movement velocity $\left(\mathrm{F}(3,38)=20.770, p<0.001, \eta^{2}=0.347\right)$ and

258

259

260

261

262

263

264

265

266

267

268

269

270

271

272

273

274

275

276

277

278

279

280

281

282

283

directional control $\left(\mathrm{F}(3,38)=75.478, p<0.001, \eta^{2}=0.659\right)$. The significant main effect of wearing experience was also determined for directional control $\left(\mathrm{F}(1,40)=5.114, p=0.029, \mathrm{\eta}^{2}=0.116\right)$. The results of post hoc analysis showed that COG movement velocity decreased significantly when wearing $3.9 \mathrm{~cm}$ HHS compared with $10.1 \mathrm{~cm}$ HHS among experienced wearers $(p=0.001,95 \%$ $\left.\mathrm{CI}=0.310^{\circ} / \mathrm{s}-1.480^{\circ} / \mathrm{s}\right)$. Experienced HHS wearers exhibited significantly higher COG directional control than inexperienced wearers when wearing $10.1 \mathrm{~cm} \mathrm{HHS}(\mathrm{t}=-3.391, p=0.002)$. Insert Table 5 here

\subsection{Functional mobility}

Table 6 illustrates that the two-way interaction $(\mathrm{HH} *$ wearing experience) was significant for FRT distance $\left(\mathrm{F}(3,38)=3.858, p=0.016, \mathrm{\eta}^{2}=0.090\right)$ and TUGT time $(\mathrm{F}(3,38)=9.883, p<0.001$, $\left.\eta^{2}=0.202\right)$. The main effect of HH was significant for FRT distance $(\mathrm{F}(3,38)=94.859, p<0.001$, $\left.\eta^{2}=0.709\right)$ and TUGT time $\left(F(3,38)=127.372, p<0.001, \eta^{2}=0.766\right)$. Significant main effect of wearing experience was also determined for FRT distance $\left(\mathrm{F}(1,40)=10.840, p=0.002, \eta^{2}=0.217\right)$ and TUGT time $\left(\mathrm{F}(1,40)=10.639, p=0.0021, \eta^{2}=0.214\right)$. With respect to the results of the pairwise comparison, generally, functional mobility decreased as $\mathrm{HH}$ increased. FRT distance was significantly shorter in 10.1 HHS than in flat shoes $(p<0.001,95 \% \mathrm{CI}=3.170-8.973 \mathrm{~cm})$, $3.9 \mathrm{~cm}(p<0.001,95 \% \mathrm{CI}=4.254-8.146 \mathrm{~cm})$, and $7 \mathrm{~cm}$ HHS $(p<0.001,95 \% \mathrm{CI}=2.675-6.225$ $\mathrm{cm})$ among experienced wearers. TUGT time showed a significant difference when wearing different HHS in experienced and inexperienced wearers. Experienced wearers performed longer FRT distance than inexperienced wearers in $3.9 \mathrm{~cm}(\mathrm{t}=-2.714, p=0.010), 7 \mathrm{~cm}(\mathrm{t}=-2.805$, $p=0.003)$ and $10.1 \mathrm{~cm}$ HHS $(\mathrm{t}=-4.524, p<0.001)$. Similarly, TUGT time in experienced wearers was significantly shorter than inexperienced HHS wearers in $3.9 \mathrm{~cm}(\mathrm{t}=3.528, p=0.010), 7 \mathrm{~cm}$ $(\mathrm{t}=3.117, p=0.003)$, and $10.1 \mathrm{~cm} \mathrm{HHS}(\mathrm{t}=3.698, p=0.001)$.

\section{Insert Table 6 here}

\subsection{Perceived stability}

The main effect of $\mathrm{HH}\left(\mathrm{F}(3,38)=26.911, p<0.001, \eta^{2}=0.415\right)$ and wearing experience 
$284\left(\mathrm{~F}(1,40)=11.517, p=0.001, \mathrm{n}^{2}=0.027\right)$ was significant for perceived stability. No significant two-

285

286

287

288

289

290

291

292

293

294

295

296

297

298

299

300

301

302

303

304

305

306

307

308

309

310

way interaction was detected on perceived stability. The perceived stability was decreased with increased HH. Specificity, the perceived stability reduced significantly in $7 \mathrm{~cm}$ HHS relative to flat shoes $(p=0.001,95 \% \mathrm{CI}=5.530-26.049)$ and $3.9 \mathrm{~cm}$ HHS $(p=0.029,95 \% \mathrm{CI}=0.940-23.060)$ among experienced wearers. The inexperienced wearers also perceived significantly decreased stability with increased HH similar to the experienced wearers (Table 6). The experienced wearers perceived significantly higher stability than inexperienced wearers in $3.9 \mathrm{~cm}(\mathrm{t}=-3.538$, $p=0.002), 7 \mathrm{~cm}(\mathrm{t}=-3.719, p=0.001)$, and $10.1 \mathrm{~cm}$ HHS $(\mathrm{t}=-2.656, p=0.011)$.

\section{Insert Table 6 here}

\section{Discussion}

The main purpose of the study is to evaluate the effects of $\mathrm{HH}$ and $\mathrm{HHS}$ wearing experience on human postural stability under dynamic perturbations. During self-initiated standing perturbations, HHS wearers exhibited decreased equilibrium and strategy scores in $10.1 \mathrm{~cm} \mathrm{HHS}$, compared with flat shoes and 3.9 and $7 \mathrm{~cm}$ HHS. Vision played a vital role in the integration of the sensory systems in the postural control process with elevated $\mathrm{HH}$. With respect to the control of the COG movement, the COG movement velocity and directional control declined in $10.1 \mathrm{~cm}$ HHS compared with flat shoes and $3.9 \mathrm{~cm}$ HHS. During external support surface perturbations, the postural latencies tended to delay with elevated $\mathrm{HH}$. Amplitude scaling increased when $\mathrm{HH}$ increased to $3.9 \mathrm{~cm}$ compared with flat shoes. Similarly, impaired functional mobility can be detected in $3.9 \mathrm{~cm}$ HHS contrary to flat shoes. However, experienced HHS wearers did not show significant higher composite equilibrium scores than novices as the authors hypothesized. No difference in the somatosensory function and postural responses under external perturbations was found between the two groups. Experienced wearers utilized higher proportion of ankle strategy and COG directional control in maintaining postural stability. They perceived higher stability and performed better functional mobility than inexperienced HHS wearers.

In SOT, decreased equilibrium and strategy scores were found in $10.1 \mathrm{~cm}$ HHS, compared with flat shoes and 3.9 and $7 \mathrm{~cm}$ shoes. The ability to integrate the sensory systems to maintain 
311 the stability of the body's equilibrium was impaired in 10.1 HHS. HHS wearers intended to use a 312 larger portion of vision than proprioception in the postural control process when wearing 10.1

$313 \mathrm{~cm}$ HHS. However, the anticipatory postural reactions from proprioceptive receptors played a 314 vital role in maintaining balance, especially in the absence of vision (Mika et al., 2016). In SOT, 315 the elevated HH may simulate an unstable condition. The sensory condition is more challenged 316 because the support surface and vision are sway referenced. Humans can increase sensory 317 weighting to vestibular and vision information for postural orientation when surrounded by these sway-referenced vision and unstable surfaces (Horak, 2006). Our study demonstrated that hip strategy was adopted more than ankle strategy by HHS wearers with increased HH under interfered conditions. With the increase in $\mathrm{HH}$, the distance of the ankle and hip joints from the line of gravity is reduced (Stefanyshyn et al., 2000). HHS wearers cannot exert torque at the ankles to rapidly move the body's COM (Horak \& Kuo, 2000; Wan et al., 2019). A higher percentage of hip strategy is used to generate a larger torque about the hip joint to realign the COG in response to higher HH (Vanicek et al., 2013). The early activation of the hip flexors may be involved in response to the translation of support surface (Horak \& Kuo, 2000). Our study's results are in line with Xiong's study, in which the hip strategy was used because the ankle strategy failed to maintain balance when wearing HHS (Hapsari \& Xiong, 2016). The ankle strategy is the first postural control strategy adopted by humans to counteract small perturbations of the COG. On the contrary, hip strategy is used in response to larger perturbations. Human often utilize the combination of ankle and hip strategies for postural correction under external perturbations. The proportion of the strategies that distributed in the postural correction is organized by central nervous system (CNS), based on somatosensory input (Shumway-Cook \& Horak, 1986). Our study showed that the HHS wearing experience had no significant effect on the overall human postural control. Human postural control is considered a complex motor skill with respect to the support surface, visual environment, and cognitive process (Shumway-Cook 336 337 \& Horak, 1986). Experienced wearers were found to adapt to walking regularity more flexibly under cognitive load than HHS novices (Schaefer \& Lindenberger, 2013). Significant different 
338 muscle efforts were exerted in HHS experts compared with novices (Stefanyshyn et al., 2000).

339 Studies have shown that wearing experience can influence the ankle ROM and muscle strength.

340 A more supinated position was found in HHS experts compared with novices. The higher ankle

341 ROM of inversion and plantarflexion might affect efficient force conduction on foot arch and

342 increase the risk of anterior talofibular ligament sprains in experienced HHS wearer (Ebbeling et

343 al., 1994; Kim et al., 2013). The long-term adaptation of the supinated position in HHS experts

344 would shorten the length of muscle fibers, decrease the amount of cross bridges of the muscle

345 fibers and disturb the function of calf muscles ultimately (Timmins et al., 2016). The muscle

346 performance of calf muscles might be affected on account of the increased concentric contraction

347 power in ankle inversion. The increased mediolateral instability would induce the changes in

348 power production owning to the habitual use of narrow heels in experts (Stefanyshyn et al.,

349 2000). In addition, the muscle performance of calf muscles may be affected on account of the

350 decreased plantarflexion torque and higher reduction on plantarflexion power in experienced

351 HHS wearers (Farrag \& Elsayed, 2016). Generally, HHS experience might further influence

352 muscle activities and cognitive processing. However, the ability to integrate the sensory systems

353 in postural control was not altered; this finding is supported by Xiong's study (Hapsari \& Xiong, 354 2016).

355 With regard to MCT, the amplitude scaling increased significantly when $\mathrm{HH}$ reach $3.9 \mathrm{~cm}$.

356 Although the composite latency was $4.06 \%$ lower in $10.1 \mathrm{HH}$ than in $3.9 \mathrm{~cm} \mathrm{HH}$, no

357 significantly delayed postural latency in response to external perturbations was found in our

358 study. Similarly, previous studies have shown no significant difference in postural reaction time

359 when wearing flip-flops, clog style Crocs, and Vibram Five-Fingers (Chander et al., 2016).

360 Footwear design characteristics may influence human postural reaction because elevated $\mathrm{HH}$ can

361 disturb the ROM of ankle joints and affect human postural control in response to forward

362 translations accordingly. When $\mathrm{HH}$ reached $3.9 \mathrm{~cm}$, the increased amplitude scaling suggested

363 that HHS wearers may alter motor output strategies to maintain postural stability under

364 perturbations. In the motor output process, the gastrocnemius medialis (GM), gastrocnemius 
365

366

367

368

369

370

371

372

373

374

375

376

377

378

379

380

381

382

383

384

385

386

387

388

389

390

391

lateralis (GL), tibialis anterior (TA), and vastus lateralis (VL) were found to exert more effort when wearing $7 \mathrm{~cm}$ HHS compared to flat shoes (Hapsari \& Xiong, 2016). The threshold of afferent discharge of muscle spindle was raised. The HHS wearers' postural control can be affected for the somatosensory alternation around the ankle and foot (Gefen et al., 2002). However, no adverse effect on postural reaction was found even in $10.1 \mathrm{~cm}$ HHS. This finding suggested that the delay of latency was often associated with neurological disorders and anatomical constraints, other than the footwear design (Redfern et al., 2001). Previous studies demonstrated that HHS can impair human balance during other extrinsic perturbations (e.g., sinusoidal oscillations and waist pulling) (Choi \& Cho, 2006; Sun et al., 2017). Sun et al. found that the COP displacement increased, and the COP trajectory transferred to the medial foot significantly during AP and ML perturbations when wearing $6.6 \mathrm{~cm}$ compared with $0.8 \mathrm{~cm} \mathrm{HH}$. However, the study did not control the shoe design and applied three types of HHS in the experiment (Sun et al., 2017). Choi and Cho compared human balance control of HHS wearers in barefoot and high-heeled posture when experiencing a waist-pull perturbation by quantifying the displacement and velocity of the COP. Results suggested that human balance control was approximately twice worse in HHS than barefoot, and the perturbation amplitude was not attributed to the participants' body weight and height (Choi \& Cho, 2006). Experienced HHS wearers exhibited no improvement in postural control under dynamic perturbations. They applied different muscle activation patterns compared with inexperienced wearers. Experienced wearers exerted significantly more muscle activities on GM and less muscular effort on VL, TA, and erector spinae than novices in SOT (Hapsari \& Xiong, 2016). During HHS walking, substantial increases in muscle fascicle strains and muscle activation were found in experienced HHS wearers compared with barefoot walking during the stance phase (Cronin et al., 2012). Experienced wearers may regulate the flexibility of the neuromuscular system to adapt to possible perturbations (e.g., walking and external perturbations) and can vary according to different HHs (Alkjær et al., 2012).

Our study investigated that the COG movement velocity and directional control in LOS 
392 significantly decreased in $10.1 \mathrm{~cm}$ compared with that in $3.9 \mathrm{~cm}$ HHS. Consistent with the 393 previous study, when $\mathrm{HH}$ increased to $10 \mathrm{~cm}$, slower COG movement velocity was observed in $39410 \mathrm{~cm}$ than in $4 \mathrm{~cm} \mathrm{HH}$ in LOS (Mika et al., 2016). The increased HH may induce the fear of 395 falling in HHS wearers. The HHS wearers manifested slow COG movement velocity, declined 396 COG excursions, and worst directional control, particularly in the forward and backward 397 directions (Hapsari \& Xiong, 2016). The experienced HHS wearers showed higher percentage of 398 directional control in $10.1 \mathrm{~cm}$ HHS. It may be due to the motor learning effects in the experienced wearer, resulting in superior ankle strategy in maintaining postural stability (Schaefer \& Lindenberger, 2013). Nonetheless, another study suggested that the increased muscular coactivation around the ankle joint could enhance joint stiffness during HHS walking. The walking balance may be improved through altered muscle activation patterns (Alkjær et al., 2012; Nielsen \& Kagamihara, 1993). The effects of muscle activation patterns on the postural control process in LOS among HHS wearers remain unclear.

The functional mobility was impaired when $\mathrm{HH}$ reached $3.9 \mathrm{~cm}$. A number of studies have shown that walking in HHS may affect neuromechanics and kinematics of the lower limbs when $\mathrm{HH}$ increased to $4 \mathrm{~cm} \mathrm{HH}$ (Naik et al., 2017). When walking in 4 and $10 \mathrm{~cm}$ HHS compared with flat shoes, the postural stability may be decreased on the account of high joint stiffness evaluated by muscle pair synchronization around the knee joint (Pratihast et al., 2018). Accordingly, the TUGT completion time was longer for impaired postural stability and reduced perceived stability, consistent with previous findings (Arnadottir \& Mercer, 2000). Our study found that the experienced HHS wearers had significantly shorter TUGT completion time and FRT distance than the novices. Long-time use of HHS has been suggested to shorten the gastrocnemius muscle fascicles and increase the Achilles tendon stiffness, thereby contributing to a restricted ankle ROM and reduced functional reach mobility (Csapo et al., 2010). Cronin et al. suggested that experienced HHS wearers may have increased muscle fascicle strains and lower limb muscle activation than inexperienced wearers during HHS walking. This finding indicates chronic 418 adaptations in muscle-tendon structure related to HHS (Cronin et al., 2012). The experienced 
419

420

421

422

423

424

425

426

427

428

429

430

431

432

433

434

435

436

437

438

439

440

441

442

443

444

445

wearers could apply altered movement strategies to increase effort on muscular control around the knee and ankle joints, so as to obtain postural stability during HHS walking. However, high muscle activities may contribute to muscle inefficiency and raised energy cost during walking, thereby leading to muscle strains, muscle fatigue, and pain (Cronin, 2014; Csapo et al., 2010; Ebbeling et al., 1994).

Although we found better functional mobility and higher perceived stability in experienced HHS wearer, no significant increase in overall postural control was detected in long-time HHS users in SOT. In functional tests, important resources, such as biomechanical constraints (e.g., strength and limits of stability), cognitive processing (e.g., learning and attention), movement strategies (e.g., anticipatory and voluntary), and sensory strategies (e.g., sensory integration and reweighting), are required for postural control. Thus, the loss of somatosensory in the foot and higher sensory weighting in vision cannot completely predict the deficiencies in functional mobility because the function depends on the aforementioned resources likewise (Horak, 2006; Horak and Kuo, 2000). In terms of $\mathrm{HH}$, we assume that the decreased perceived comfort and loss of joint position may lead to low perceived stability, compromising functional mobility accordingly (Hong et al., 2005; Lee \& Hong, 2005).

The limitation of the study is that the results may not be extrapolated to all HHS populations from different ages and health statuses, considering that we only recruited healthy young females in our study. Besides, the neuromuscular mechanism of postural control in HHS wearers is still unknown. The effects of HH and long-term use of HHS on lower limb muscle activities, muscle coordination, and Hoffmann reflex need to be further studied to elucidate how CNS controls motor output in the postural control process. Furthermore, to provide evidence-based information for clinicians, more cohort studies can be conducted to explore the relationship between wearing experience and HHS-related injuries such as metatarsalgia and ankle sprain.

\section{Conclusions}

Perceived stability and functional mobility decreased when wearing HHS. The vision system had high weight in maintaining postural stability when $\mathrm{HH}$ increased to $10.1 \mathrm{~cm}$. During 
446

447

448

449

450

451

452

453

454

455

456

457

458

459

460

461

462

463

464

465

466

467

468

469

470

471

472

dynamic perturbations, higher percentage of ankle strategies and motor control strategies was exhibited when wearing $3.9 \mathrm{~cm}$ HHS compared with flat shoes. In terms of HHS experience, experienced HHS wearers used higher proportion of ankle strategy and COG directional control in postural control than novices. In addition, experienced wearers perceived higher postural stability and showed better functional mobility. It is recommended that on evaluating the postural stability of HHS wearers, sensory organization ability, ankle strategy and COG directional control could be considered to be useful in developing a safety system and prevent HHS wearers from falling.

\section{Acknowledgment}

Yiyang Chen contributed to conceptualization, methodology, formal analysis, investigation, and writing - Original Draft. Lin Wang contributed to conceptualization, methodology, and supervision. Jing Xian Li participated in conceptualization and writing - Review \& Editing. The authors appreciate the kind participation of all the subjects.

\section{Reference}

American Podiatric Medical Association. 2003. High-Heel Survey, 2003. Available at: www.apma.org/s_apma/doc.asp?CID=385\&DID= 17112 (accessed 15 January 2014)

Alkjær T, Raffalt P, Petersen NC, Simonsen EB. 2012. Movement Behavior of High-Heeled Walking: How Does the Nervous System Control the Ankle Joint during an Unstable Walking Condition? PLOS ONE 7(5):e37390

Arnadottir SA, Mercer VS. 2000. Effects of Footwear on Measurements of Balance and Gait in Women Between the Ages of 65 and 93 Years. Physical Therapy 80(1):17-27

Chander H, Morris CE, Wilson SJ, Garner JC, Wade C. 2016. Impact of alternative footwear on human balance. Footwear Science 8(3):165-174

Chien HL, Lu TW, Liu MW. 2013. Control of the motion of the body's center of mass in relation to the center of pressure during high-heeled gait. Gait \& Posture 38(3):391-396 
473 Chien HL, Lu TW, Liu MW. 2014. Effects of long-term wearing of high-heeled shoes on the 474 control of the body's center of mass motion in relation to the center of pressure during 475 walking. Gait \& Posture 39(4):1045-1050

476

477

478

479

480

481

482

483

484

485

486

Choi HK, Cho WH. 2006. The Effects of High-Heeled Posture on COP Kinematics and Muscle Fatigue during the Balance Control of Human Body. Key Engineering Materials 321323:1119-1122

Cronin NJ. 2014. The effects of high heeled shoes on female gait: A review. Journal of Electromyography and Kinesiology 24(2):258-263

Cronin NJ, Barrett RS, Carty CP. 2012. Long-term use of high-heeled shoes alters the neuromechanics of human walking. Journal of Applied Physiology 112(6):1054-1058

Csapo R, Maganaris CN, Seynnes OR, Narici MV. 2010. On muscle, tendon and high heels. Journal of Experimental Biology 213(15):2582-2588

Dickin DC. 2010. Obtaining Reliable Performance Measures on the Sensory Organization Test: Altered Testing Sequences in Young Adults. Clinical Journal of Sport Medicine : Official Journal of the Canadian Academy of Sport Medicine 20(4):278-285

Ebbeling CJ, Hamill J, Crussemeyer JA. 1994. Lower Extremity Mechanics and Energy Cost of Walking in High-Heeled Shoes. Journal of Orthopaedic \& Sports Physical Therapy 19(4):190-196

Farrag A, Elsayed W. 2016. Habitual Use of High-Heeled Shoes Affects Isokinetic Soleus Strength More Than Gastrocnemius in Healthy Young Females. Foot and Ankle International 37(9):1008-1016

Ford-smit CD, Wyman JF, Elswick RK, Fernandez T, Newton RA. 1995. Test-Retest Reliability of The Sensory Organization Test in Noninstitutionalized Older Adults. Archives of Physical Medicine and Rehabilitation 76(1):77-81

Gefen A, Megido-Ravid M, Itzchak Y, Arcan M. 2002. Analysis of muscular fatigue and foot stability during high-heeled gait. Gait \& Posture 15(1):56-63

Gerber SB, Costa RV, Grecco LAC, Pasini H, Marconi NF, Oliveira CS. 2012. Interference of 
500

501

502

503

504

505

506

507

508

509

510

511

512

513

514

515

516

517

518

519

520

521

522

523

524

525

526

high-heeled shoes in static balance among young women. Human Movement Science 31(5):1247-1252

Hapsari VD, Xiong S. 2016. Effects of high heeled shoes wearing experience and heel height on human standing balance and functional mobility. Ergonomics 59(2):249-264

Harro CC, Garascia C. 2019. Reliability and Validity of Computerized Force Platform Measures of Balance Function in Healthy Older Adults. Journal of Geriatric Physical Therapy 42(3):57-66

Hebert JR, Manago MM. 2017. Reliability and validity of the computerized dynamic posturography sensory organization test in people with multiple sclerosis. International Journal of MS Care 19(3):151-157

Hong WH, Lee YH, Chen HC, Pei YC, Wu CY. 2005. Influence of Heel Height and Shoe Insert on Comfort Perception and Biomechanical Performance of Young Female Adults During Walking. Foot \& Ankle International 26(12):1042-1048

Horak FB. 2006. Postural orientation and equilibrium: What do we need to know about neural control of balance to prevent falls? Age and Ageing 35(SUPPL.2):7-11

Horak FB, Kuo A. 2000. Postural Adaptation for Altered Environments, Tasks, and Intentions In: Winters J.M, Crago PE, ed. Biomechanics and Neural Control of Posture and Movement. New York: Springer, 267-281

Jayakaran P, Johnson GM, Sullivan SJ. 2011. Test-Retest Reliability of the Sensory Organization Test in Older Persons With a Transtibial Amputation. The Journal of Injury, Function, and Rehabilitation 3(8):723-729

Kermani M, Ghasemi M, Rahimi A, Khademi-Kalantari K, Akbarzadeh-Bghban A. 2018. Electromyographic changes in muscles around the ankle and the knee joints in women accustomed to wearing high-heeled or low-heeled shoes. Journal of Bodywork and Movement Therapies 22(1):129-133

Kim Y, Lim JM, Yoon B. 2013. Changes in ankle range of motion and muscle strength in habitual wearers of high-heeled shoes. Foot and Ankle International 34(3):414-419 
527 Ko PH, Hsiao TY, Kang JH, Wang TG, Shau YW, Wang CL. 2009. Relationship between 528 plantar pressure and soft tissue strain under metatarsal heads with different heel heights.

$529 \quad$ Foot and Ankle International 30(11):1111-1116

530 Lee YH, Hong WH. 2005. Effects of shoe inserts and heel height on foot pressure, impact force, 531 and perceived comfort during walking. Applied Ergonomics 36(3):355-362

532 Luximon Y, Cong Y, Luximon A, Zhang M. 2015. Effects of heel base size, walking speed, and 533 slope angle on center of pressure trajectory and plantar pressure when wearing high-heeled

Mancini M, Horak FB. 2010. The relevance of clinical balance assessment tools to differentiate shoes. Human Movement Science 41:307-319

Mika A, Oleksy Ł, Kielnar R, Świerczek M. 2016. The influence of high- and low-heeled shoes on balance in young women. Acta of Bioengineering and Biomechanics 18(3):97-103

Naik GR, Al-Ani A, Gobbo M, Nguyen HT. 2017. Does Heel Height Cause Imbalance during Sit-to-Stand Task: Surface EMG Perspective. Frontiers in Physiology 8:626

Nielsen J, Kagamihara Y. 1993. The regulation of presynaptic inhibition during co-contraction of antagonistic muscles in man. The Journal of Physiology 464(1):575-593

Pratihast M, Al-Ani A, Chai R, Su S, Naik G. 2018. Changes in lower limb muscle synchronisation during walking on high-heeled shoes. Healthcare Technology Letters $5(6): 236-238$

Rahimi A, Sayah A, Hosseini SM, Baghban AA. 2017. Studying the Plantar Pressure Patterns in Women Adapted to High-Heel Shoes during Barefoot Walking. Journal of Clinical Physiotherapy Research 2(2):70-74

Redfern MS, Cham R, Gielo-Perczak K, Grönqvist R, Hirvonen M, Lanshammar H, Marpet M, Pai CYC, Powers C. 2001. Biomechanics of slips. Ergonomics 44(13):1138-1166

Schaefer S, Lindenberger U. 2013. Thinking While Walking: Experienced High-Heel Walkers Flexibly Adjust Their Gait. Frontiers in Psychology 4:316

Schoppen T, Boonstra A, Groothoff JW, Vties J. 1999. The Timed “Up and Go ” Test : 
554

555

556

557

558

559

560

561

562

563

564

565

566

567

568

569

570

571

572

573

574

575

576

577

578

579

580

Reliability and Validity in Persons With Unilateral Lower Limb Amputation. Archives of Physical Medicine and Rehabilitation 80(7):825-828

Schroeder J, Hollander K. 2018. Effects of high-heeled footwear on static and dynamic pelvis position and lumbar lordosis in experienced younger and middle-aged women. Gait \& Posture 59:53-57

Shumway-Cook A, Horak FB. 1986. Assessing the Influence of Sensory Interaction on Balance. Physical Therapy 66(10):1548-1550

Simonsen EB, Svendsen MB, Nørreslet A, Baldvinsson HK, Heilskov-Hansen T, Larsen PK, Alkjær T, Henriksen M. 2012. Walking on High Heels Changes Muscle Activity and the Dynamics of Human Walking Significantly. Journal of Applied Biomechanics 28(1):20-28

Stefanyshyn DJ, Nigg BM, Fisher V, O’Flynn B, Li W. 2000. The influence of high heeled shoes on kinematics, kinetics, and muscle EMG of normal female gait. Journal of Applied Biomechanics 16(3):309-319

Sun D, Gu Y, Mei Q, Shao Y, Sun J, Fernandez J. 2017. Effect of Heel Heights on Female Postural Control During Standing on a Dynamic Support Surface With Sinusoidal Oscillations. Journal of Motor Behavior 49(3):281-287

Timmins RG, Bourne MN, Shield AJ, Williams MD, Lorenzen C, Opar DA. 2016. Short biceps femoris fascicles and eccentric knee flexor weakness increase the risk of hamstring injury in elite football (soccer): A prospective cohort study. British Journal of Sports Medicine 50(24):1524-1535

Truszczyńska A, Trzaskoma Z, Stypińska Z, Drzał-Grabiec J, Tarnowski A. 2019. Is static balance affected by using shoes of different height? Biomedical Human Kinetics 8(1):137144

Tsang WW, Wong VS, Fu SN, Hui-chan CW. 2004. Tai Chi Improves Standing Balance Control Under Reduced or Conflicting Sensory Conditions. Archives of Physical Medicine and Rehabilitation 85(1):129-137

Vanicek N, King SA, Gohil R, Chetter IC, Coughlin PA. 2013. Computerized dynamic 
581 posturography for postural control assessment in patients with intermittent claudication.

$582 \quad$ Journal of Visualized Experiments : JoVE 82:1-9

583 Wan FKW, Yick KL, Yu WWM. 2019. Effects of heel height and high-heel experience on foot $584 \quad$ stability during quiet standing. Gait \& Posture 68:252-257

585 Winter D. 1995. Human balance and posture control during standing and walking. Gait \& $586 \quad$ Posture 3(4):193-214

587 Yin L, Wang L. 2020. Acute Effect of Kinesiology Taping on Postural Stability in Individuals 588 With Unilateral Chronic Ankle Instability. Frontiers in Physiology 11:1-8

589 Zifchock RA, Theriot C, Hillstrom HJ, Song J, Neary M. 2017. The Relationship Between Arch $590 \quad$ Height and Arch Flexibility: A Proposed Arch Flexibility Classification System for the 591 Description of Multidimensional Foot Structure. Journal of the American Podiatric Medical 592 Association 107(2):119-123 
Table $\mathbf{1}$ (on next page)

Six testing conditions of SOT. 
1 Table 1:

2 Six testing conditions of SOT.

\begin{tabular}{lllll}
\hline Condition & Eyes & Support Surface & Visual Surroundings & Anticipated Sensory Systems \\
\hline 1 & Open & Fixed & Fixed & Somatosensory \\
2 & Closed & Fixed & Fixed & Somatosensory \\
3 & Open & Fixed & Sway referenced & Somatosensory \\
4 & Open & Sway referenced & Fixed & Vision and vestibular \\
5 & Closed & Sway referenced & Fixed & Vestibular \\
6 & Open & Sway referenced & Sway referenced & Vestibular \\
\hline
\end{tabular}

3 
Table 2 (on next page)

Demographic data of the participants.

Note: BMI, Body Mass Index; AHF, Arch Height Flexibility; *, inexperienced vs. experienced HHS wearers, $p<0.05$. 
1 Table 2:

2 Demographic data of the participants.

\begin{tabular}{lll}
\hline & $\begin{array}{l}\text { Inexperienced HHS wearers } \\
(\mathrm{N}=21)\end{array}$ & $\begin{array}{l}\text { Experienced HHS wearers } \\
(\mathrm{N}=20)\end{array}$ \\
\hline Age (years) & $25.05 \pm 1.63$ & $23.05 \pm 2.24$ \\
Height $(\mathrm{cm})$ & $1.63 \pm 0.05$ & $1.63 \pm 0.05$ \\
Weight $(\mathrm{Kg})$ & $57.51 \pm 7.87$ & $56.33 \pm 6.94$ \\
BMI $\left(\mathrm{Kg} / \mathrm{m}^{2}\right)$ & $21.62 \pm 2.35$ & $21.09 \pm 2.62$ \\
Foot length $10 \%$ weightbearing $(\mathrm{mm})$ & $231.43 \pm 8.50$ & $231.25 \pm 9.50$ \\
Foot length 90\% weightbearing $(\mathrm{mm})$ & $234.95 \pm 8.27$ & $235.15 \pm 10.25$ \\
AHF $(\mathrm{mm} / \mathrm{kN})$ & $0.90 \pm 0.05$ & $0.70 \pm 0.04$ \\
HHS wearing frequency (hours/week) & $2.19 \pm 4.61$ & $28.33 \pm 10.13 *$ \\
\hline
\end{tabular}

3 Note: BMI, Body Mass Index; AHF, Arch Height Flexibility; *, inexperienced vs. experienced 4 HHS wearers, $p<0.05$. 


\section{Table 3(on next page)}

Comparison of outcome measures (means \pm SD) in SOT for four HHS in inexperienced and experienced groups.

SOM, somatosensory score; VIS, visual score; VEST, vestibular score; *, Inexperienced vS. experienced HHS wearers, $p<0.05$. 
1 Table 3:

2 Comparison of outcome measures (means \pm SD) in SOT for four HHS in inexperienced and experienced groups. Inexperienced HHS wearers ( $\mathrm{N}=21)$

\section{Equilibrium score}

$\begin{array}{llll}93.02 \pm 3.72 & 93.40 \pm 2.82 & 92.46 \pm 3.36 & 91.52 \pm 2.40 \\ 90.76 \pm 2.58 & 91.20 \pm 4.21 & 89.44 \pm 4.24 & 87.86 \pm 4.25 \\ 89.89 \pm 4.42 & 89.91 \pm 3.99 & 88.86 \pm 4.59 & 86.52 \pm 4.34 \\ 85.35 \pm 10.46 & 88.19 \pm 9.99 & 87.78 \pm 7.44 & 89.95 \pm 3.40 \\ 80.11 \pm 10.37 & 79.56 \pm 9.39 & 81.14 \pm 6.44 & 80.56 \pm 4.78 \\ 72.97 \pm 10.87 & 76.81 \pm 9.46 & 77.25 \pm 9.37 & 80.90 \pm 5.23 \\ 83.52 \pm 7.35 & 84.86 \pm 5.94 & 84.86 \pm 5.70 & 84.52 \pm 3.16\end{array}$

$91.37 \pm 2.76$

$93.57 \pm 2.03$

\section{$92.80 \pm 2.61$}

$91.55 \pm 2.59$

$90.83 \pm 1.71$

$91.25 \pm 3.21$

$91.08 \pm 2.76$

$89.57 \pm 4.01$

$89.62 \pm 6.08$

$90.00 \pm 4.83$

$81.82 \pm 7.81 \quad 79.62 \pm 9.01$

$80.78 \pm 5.45$

$6.70 \pm 12.27 \quad 75.65 \pm 9.80$

$79.10 \pm 11.12$

$85.85 \pm 6.33 \quad 85.20 \pm 4.70$

$85.95 \pm 4.84$

$95.58 \pm 1.34 \quad 95.90 \pm 1.18$

$94.78 \pm 1.64$

$95.30 \pm 1.11 *$

$94.92 \pm 1.57$

$94.98 \pm 1.73$

$93.92 \pm 1.39$

$93.90 \pm 2.34$

$93.40 \pm 2.82 \quad 92.46 \pm 3.36 \quad 91.52 \pm 2.40$

$94.17 \pm 2.01$

$91.20 \pm 4.21 \quad 89.44 \pm 4.24 \quad 87.86 \pm 4.25$

$90.58 \pm 2.44$

$95.37 \pm 1.11 *$

$94.22 \pm 2.03$

$87.22 \pm 2.76$

$90.70 \pm 2.78$

$90.03 \pm 2.30$

$85.05 \pm 4.37$

$88.19 \pm 9.99 \quad 87.78 \pm 7.44 \quad 89.95 \pm 3.40$

$85.50 \pm 5.02$

$84.70 \pm 3.42$

$86.87 \pm 3.55$

$87.10 \pm 2.54 * \quad 85.27 \pm 5.16$

$97.90 \pm 2.29$

$98.05 \pm 1.82$

$98.05 \pm 2.39$

$96.40 \pm 3.95 \quad 0.031$

0.450

\begin{tabular}{|c|c|c|c|c|c|c|c|c|c|c|c|}
\hline SOM & $97.86 \pm 3.14$ & $97.62 \pm 3.32$ & $96.81 \pm 3.09$ & $96.19 \pm 3.93$ & $97.90 \pm 2.29$ & $98.05 \pm 1.82$ & $98.05 \pm 2.39$ & $96.40 \pm 3.95$ & 0.031 & 0.450 & 0.756 \\
\hline VIS & $91.71 \pm 9.56$ & $94.48 \pm 9.42$ & $94.90 \pm 6.06$ & $98.57 \pm 3.60$ & $95.20 \pm 9.05$ & $95.95 \pm 6.08$ & $97.10 \pm 4.12$ & $97.95 \pm 4.37$ & 0.010 & 0.247 & 0.484 \\
\hline VEST & $86.10 \pm 10.24$ & $85.10 \pm 9.08$ & $87.62 \pm 5.95$ & $88.24 \pm 4.89$ & $87.45 \pm 7.57$ & $85.10 \pm 9.57$ & $87.15 \pm 4.98$ & $89.20 \pm 6.70$ & 0.097 & 0.781 & 0.872 \\
\hline
\end{tabular}


Table 4 (on next page)

Comparison of outcome measures (means \pm SD) in MCT for four HHS in inexperienced and experienced groups.

COMP, composite score; B, backward; F, forward; S, small; M, medium; L, large; *, inexperienced vs. experienced HHS wearers, $p<0.05$. 
1 Table 4:

2 Comparison of outcome measures (means \pm SD) in MCT for four HHS in inexperienced and experienced groups. Inexperienced HHS wearers $(\mathrm{N}=21) \quad$ Experienced HHS wearers $(\mathrm{N}=20)$

$0.8 \mathrm{~cm}$

$p$ values

$0.8 \mathrm{~cm}$

$3.9 \mathrm{~cm}$

$10.1 \mathrm{~cm}$

$3.9 \mathrm{~cm}$

$7 \mathrm{~cm}$

$10.1 \mathrm{~cm}$

Within

Between Two-way

Latency COMP (milliseconds)

$\begin{array}{llll}128.39 \pm 8.27 & 128.39 \pm 7.82 & 126.50 \pm 5.29 & 126.39 \pm 4.98\end{array}$

$131.90 \pm 11.14 \quad 134.25 \pm 15.11$

$129.20 \pm 9.02$

$128.80 \pm 7.30$ groups

groups interaction

\section{Amplitude scaling}

\begin{tabular}{|c|c|c|c|c|c|c|c|c|c|c|c|}
\hline \multirow[t]{3}{*}{$\mathrm{B}$} & $1.72 \pm 1.02$ & $2.28 \pm 1.49$ & $2.44 \pm 1.46$ & $2.83 \pm 1.15$ & $1.80 \pm 1.47$ & $1.75 \pm 1.12$ & $2.35 \pm 1.63$ & $3.00 \pm 1.56$ & $<0.001$ & 0.759 & 0.579 \\
\hline & $4.06 \pm 2.24$ & $4.61 \pm 2.89$ & $5.06 \pm 2.36$ & $4.61 \pm 1.88$ & $3.20 \pm 1.94$ & $4.10 \pm 2.29$ & $4.40 \pm 2.09$ & $4.45 \pm 2.24$ & 0.015 & 0.359 & 0.798 \\
\hline & $6.33 \pm 2.95$ & $6.78 \pm 3.34$ & $6.67 \pm 3.12$ & $6.78 \pm 2.44$ & $4.90 \pm 2.77$ & $5.65 \pm 3.00$ & $5.65 \pm 2.41$ & $6.50 \pm 2.31$ & 0.082 & 0.359 & 0.798 \\
\hline S & $2.28 \pm 1.71$ & $2.78 \pm 1.52$ & $3.28 \pm 1.74$ & $3.89 \pm 1.32$ & $2.05 \pm 1.15$ & $1.90 \pm 1.02$ & $2.25 \pm 1.41$ & $3.45 \pm 1.64$ & $<0.001$ & 0.089 & 0.314 \\
\hline M & $4.39 \pm 1.97$ & $5.44 \pm 1.85$ & $5.78 \pm 1.80$ & $6.94 \pm 1.86$ & $3.70 \pm 1.81$ & $4.90 \pm 2.29$ & $5.15 \pm 2.41$ & $6.55 \pm 2.80$ & $<0.001$ & 0.337 & 0.970 \\
\hline $\mathrm{L}$ & $6.83 \pm 2.33$ & $7.44 \pm 2.59$ & $7.78 \pm 0.56$ & $8.44 \pm 2.18$ & $5.65 \pm 2.76$ & $7.10 \pm 2.69$ & $8.15 \pm 3.10$ & $8.20 \pm 3.02$ & $<0.001$ & 0.622 & 0.328 \\
\hline
\end{tabular}

3 Note: COMP, composite score; B, backward; F, forward; S, small; M, medium; L, large; *, inexperienced vs. experienced HHS

4 wearers, $p<0.05$. 


\section{Table 5 (on next page)}

Comparison of outcome measures (means \pm SD) in LOS for four HHS in inexperienced and experienced groups.

Note: COG, center of gravity; ${ }^{*}$, Inexperienced vs. experienced HHS wearers, $p<0.05$. 
1 Table 5:

2 Comparison of outcome measures (means \pm SD) in LOS for four HHS in inexperienced and experienced groups. Inexperienced HHS wearers ( $\mathrm{N}=21) \quad$ Experienced HHS wearers $(\mathrm{N}=20)$

\begin{tabular}{|c|c|c|c|c|c|c|c|c|c|c|}
\hline $0.8 \mathrm{~cm}$ & $3.9 \mathrm{~cm}$ & $7 \mathrm{~cm}$ & $10.1 \mathrm{~cm}$ & $0.8 \mathrm{~cm}$ & $3.9 \mathrm{~cm}$ & $7 \mathrm{~cm}$ & $10.1 \mathrm{~cm}$ & $\begin{array}{l}\text { Within } \\
\text { groups }\end{array}$ & $\begin{array}{c}\text { Between } \\
\text { groups }\end{array}$ & $\begin{array}{l}\text { Two-way } \\
\text { interaction }\end{array}$ \\
\hline $4.86 \pm 1.79$ & $4.46 \pm 1.44$ & $4.34 \pm 1.39$ & $3.58 \pm 1.16$ & $85.85 \pm 6.33$ & $5.30 \pm 1.60$ & $5.20 \pm 1.51$ & $4.84 \pm 1.45$ & $<0.001$ & 0.155 & 0.659 \\
\hline $\begin{array}{c}\text { ontrol (\%) } \\
82.33 \pm 5.62\end{array}$ & $82.00 \pm 6.83$ & $77.67 \pm 8.48$ & $68.62 \pm 9.74$ & $84.45 \pm 3.98$ & $84.95 \pm 3.20$ & $80.20 \pm 5.65$ & $77.45 \pm 6.53 *$ & $<0.001$ & 0.029 & $<0.001$ \\
\hline
\end{tabular}

3 Note: COG, center of gravity; *, Inexperienced vs. experienced HHS wearers, $p<0.05$. 


\section{Table 6(on next page)}

Comparison of outcome measures (means \pm SD) in functional mobility test and perceived stability for four HHS in inexperienced and experienced groups.

Note: FRT, functional research test; TUGT, time up and go test; VAS, visual analog scale; *, Inexperienced vs. experienced HHS wearers, $\mathrm{p}<0.05$. 
1 Table 6:

2 Comparison of outcome measures (means \pm SD) in functional mobility test and perceived stability for four HHS in

3 inexperienced and experienced groups.

Inexperienced HHS wearers $(\mathrm{N}=21)$

\begin{tabular}{|c|c|c|c|c|c|c|c|c|c|c|c|}
\hline & $0.8 \mathrm{~cm}$ & $3.9 \mathrm{~cm}$ & $7 \mathrm{~cm}$ & $10.1 \mathrm{~cm}$ & $0.8 \mathrm{~cm}$ & $3.9 \mathrm{~cm}$ & $7 \mathrm{~cm}$ & $10.1 \mathrm{~cm}$ & $\begin{array}{l}\text { Within } \\
\text { groups }\end{array}$ & $\begin{array}{c}\text { Between } \\
\text { groups }\end{array}$ & $\begin{array}{l}\text { Two-way } \\
\text { interaction }\end{array}$ \\
\hline \multicolumn{12}{|l|}{ FRT (cm) } \\
\hline & $33.71 \pm 4.50$ & $32.01 \pm 4.86$ & $30.48 \pm 4.39$ & $23.81 \pm 3.85$ & $36.43 \pm 4.79$ & $36.29 \pm 5.25 *$ & $34.54 \pm 4.87 *$ & $30.09 \pm 5.00 *$ & $<0.001$ & 0.002 & 0.016 \\
\hline \multicolumn{12}{|l|}{ TUGT (s) } \\
\hline & $6.59 \pm 1.27$ & $7.24 \pm 1.06$ & $7.64 \pm 1.38$ & $68.62 \pm 9.74$ & $5.97 \pm 0.74$ & $6.24 \pm 0.72 *$ & $6.53 \pm 0.82 *$ & $7.35 \pm 1.10 *$ & $<0.001$ & 0.002 & $<0.001$ \\
\hline \multicolumn{12}{|c|}{ VAS (0-100) } \\
\hline & $74.71 \pm 32.31$ & $71.00 \pm 23.63$ & $58.48 \pm 19.10$ & $34.43 \pm 24.05$ & $80.40 \pm 30.76$ & $90.35 \pm 8.16^{*}$ & $77.75 \pm 13.44 *$ & $52.65 \pm 19.51 *$ & $<0.001$ & 0.001 & 0.351 \\
\hline
\end{tabular}

4 Note: FRT, functional research test; TUGT, time up and go test; VAS, visual analog scale; *, Inexperienced vs. experienced HHS

5 wearers, $p<0.05$.

6 
Figure 1

(A) Size of the heel base and (B) experimental shoes with different $\mathrm{HH}$.

$12.5 * 12.0 \mathrm{~mm}$

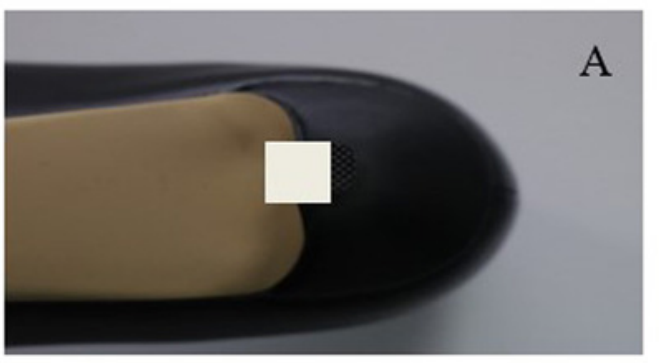

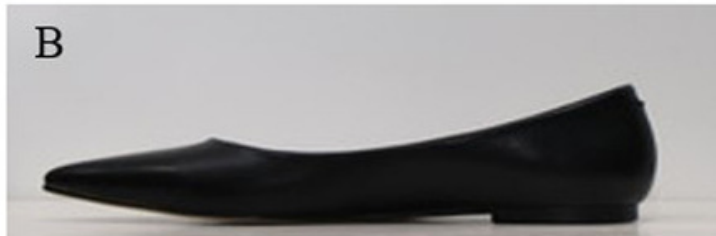

C

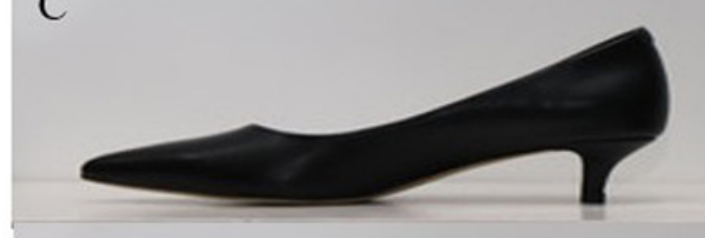

D

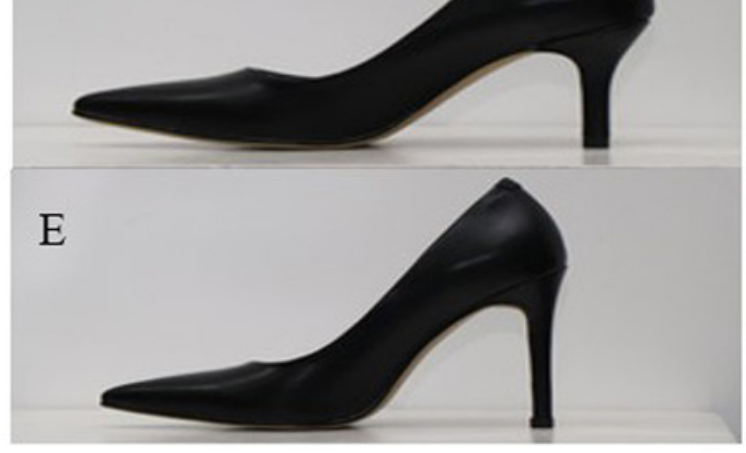

$0.8 \mathrm{~cm}$

$3.9 \mathrm{~cm}$

$7.0 \mathrm{~cm}$

$10.1 \mathrm{~cm}$ 


\section{Figure 2}

(A) Experimental setup and (B) the illustration of support surface translation in MCT.

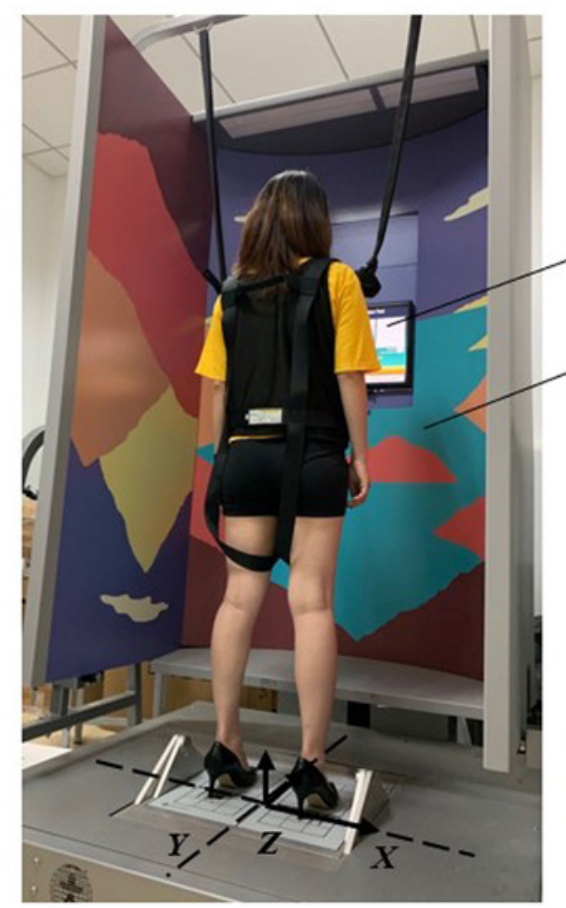

A
Computerized

Display

Moveable

Visual Surround

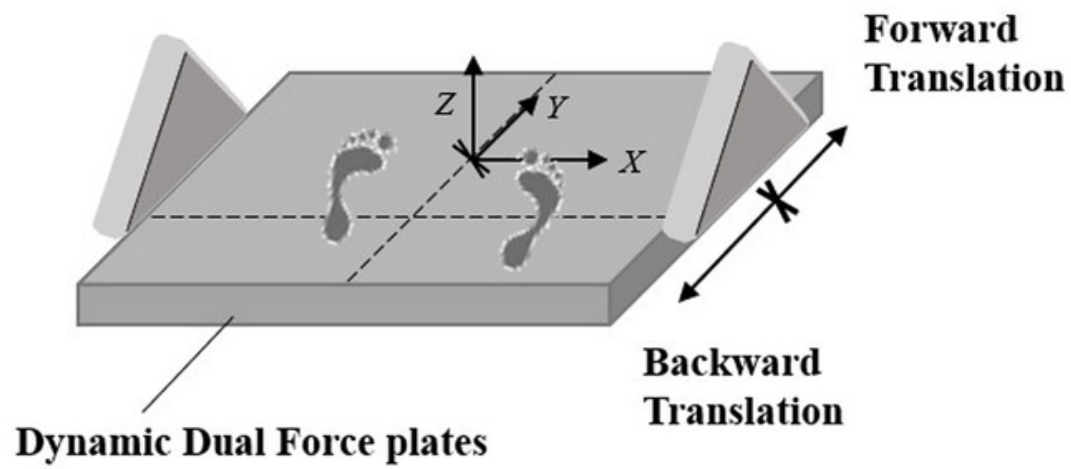

$\mathrm{B}$ 